Journal for ImmunoTherapy of Cancer

\section{Pembrolizumab for patients with leptomeningeal metastasis from solid tumors: efficacy, safety, and cerebrospinal fluid biomarkers}

To cite: Naidoo J, Schreck KC, Fu W, et al. Pembrolizumab for patients with leptomeningeal metastasis from solid tumors: efficacy, safety, and cerebrospinal fluid biomarkers. Journal for ImmunoTherapy of Cancer 2021;9:e002473. doi:10.1136/jitc-2021-002473

- Additional supplemental material is published online only. To view, please visit the journal online (http://dx.doi.org/10. 1136/jitc-2021-002473).

Accepted 02 June 2021

Check for updates

(c) Author(s) (or their employer(s)) 2021. Re-use permitted under CC BY-NC. No commercial re-use. See rights and permissions. Published by BMJ.

For numbered affiliations see end of article.

Correspondence to Dr Jarushka Naidoo jnaid001@jhmi.edu

\section{ABSTRACT}

Background The benefit of immune checkpoint inhibitors (ICls) in patients with leptomeningeal metastases (LMM) is unknown.

Methods We undertook a phase II trial of pembrolizumab in patients with LMM from solid tumors. Eligible patients had radiologic/cytologic LMM and Eastern Cooperative Oncology Group performance status 0-1. Pembrolizumab was administered intravenously at $200 \mathrm{mg}$ q3W until disease progression/unacceptable toxicity. The primary endpoint was central nervous system (CNS) response after four cycles, defined radiologically/cytologically/clinically. Serial cerebrospinal fluid (CSF) was assessed for tumorderived DNA (t-DNA) aneuploidy and cytokines.

Results Thirteen of a planned 16 patients were treated between April 2017 and December 2019. The study closed early for poor accrual. Median age was 57 years (range: 22-79). Sixty-two percent of patients had tumors not traditionally ICl-responsive (hormone-receptor (HR)positive breast carcinoma $=39 \%$; high-grade glioma $=23 \%$ ), while $38 \%$ had ICl-responsive tumors (non-small cell lung cancer $(\mathrm{NSCLC})=23 \%$, head and neck carcinoma $=8 \%$, cutaneous squamous carcinoma $(\mathrm{CSC})=8 \%$ ). CNS response was observed in $38 \%$ of patients at 12 weeks (95\% Cl $13.9 \%$ to $68.4 \%$ ) by pre-defined criteria and LM-RANO, and 2 achieved durable complete responses (CSC $=1$, overall survival $(\mathrm{OS}) 3+$ years; $\mathrm{NSCLC}=1, \mathrm{OS}$ 9 months). Median CNS progression-free survival and OS was 2.9 months ( $95 \% \mathrm{Cl} 1.3$ to NR) and 4.9 months (95\% Cl 3.7 to NR), respectively. Grade 3+ treatmentrelated adverse events occurred in $15 \%$ of patients. Sensitivity for LMM detection by t-DNA and cytopathology was $84.6 \%(95 \% \mathrm{Cl} 54.6 \%$ to $98.1 \%)$ and $53.9 \%(95 \% \mathrm{Cl}$ $25.1 \%$ to $80.8 \%$ ), respectively. Pre-therapy and on-therapy CSF cytokine analysis demonstrated complete responders clustered together.

Conclusions Pembrolizumab conferred a 38\% CNS response rate in patients with $\mathrm{LMM}$, a tolerable safety profile, and deep responses in selected patients with ICIresponsive tumors. CSF t-DNA may be sensitive for LMM detection, and immunologic subsets of CNS response warrant further study.

Trial registration number NCT03091478

\section{INTRODUCTION}

Immune checkpoint inhibitors (ICIs) benefit selected patients with brain metastases $(\mathrm{BM}) .^{12}$ In patients with metastatic melanoma, treatment with ipilimumab ${ }^{3}$ or the combination of ipilimumab and nivolumab resulted in improvements in central nervous system (CNS) response and survival. ${ }^{4}$ In patients with BM from either melanoma or PD-L1+ $(>1 \%)$ non-small cell lung cancer (NSCLC), ${ }^{5}$ pembrolizumab led to CNS responses in a phase II trial. While patients with BM have been included in studies of ICIs, patients with leptomeningeal metastasis (LMM) have largely been excluded from these trials owing to poor patient prognosis, except for one study in melanoma. ${ }^{4}$ There remains a paucity of treatment options for patients with LMM from solid tumors. In patients with LMM from breast cancer, local radiation therapy (RT) for symptomatic control \pm high-dose systemic or intrathecal chemotherapy may be used. ${ }^{78}$ In patients with LMM from oncogene-addicted tumors with targeted treatment options (eg, osimertinib in EGFR-mutant $\mathrm{NSCLC}^{9}$ ), CNS responses and improved survival outcomes occur. However, most patients with LMM have poor outcomes due to a lack of treatments that are effective both intracranially and extracranially. While there has been one published study demonstrating a higher 3-month 
survival rate from pembrolizumab for patients with LMM from solid tumors mainly in those with breast cancer, ${ }^{10}$ it is unknown whether anti-PD(L) 1 ICIs may lead to CNS responses in those with LMM from solid tumors. In addition, biomarkers of ICI response in this subset of patients have not been elucidated.

Thus, we proposed a phase II trial of pembrolizumab in patients with LMM from any solid tumor. Our primary objective was to determine if pembrolizumab led to CNS response in LMM. Secondary objectives were to determine if pembrolizumab led to improvements in CNS progression-free survival (PFS), overall survival (OS), and acceptable safety, compared with historic data. We also sought to identify whether tumor-derived DNA (t-DNA) shed into the cerebrospinal fluid (CSF) and CSF immunologic features would be detectable in patients with LMM, and correlate with CNS response or survival.

\section{PATIENTS AND METHODS \\ Patients}

Eligible patients were $\geq 18$ years old with LMM from a pathologically confirmed solid tumor malignancy. In this study, LMM was defined as either radiologically measurable LMM on contrast-enhanced MRI ( $>3 \mathrm{~mm}$ lesion) or cytologically-detectable LMM by CSF sampling. Patients with CNS metastases but without LMM were not eligible. Measurable disease outside the CNS was not a requirement for study enrollment. All potentially eligible patients were discussed at a multidisciplinary neuro-oncology conference comprising $\geq 2$ medical oncologists, a radiation oncologist, neurologist, and neuro-radiologist to confirm the diagnosis of LMM and suitability for enrollment. All enrolled patients had an Eastern Cooperative Oncology Group (ECOG) performance status (PS) of 0 or 1 , and adequate organ function (absolute neutrophil count $\geq 1500 \mu \mathrm{L}$; platelets $\geq 100,00 / \mu \mathrm{L} ; \mathrm{Hb} \geq 9 \mathrm{~g} / \mathrm{dL}$; serum creatinine $\leq 1.5$ times upper limit of normal, serum total bilirubin $\leq 1.5$ times upper limit of normal, AST and ALT $\leq 2.5$ times upper limit of normal, albumin $\geq 2.5 \mathrm{mg}$ / $\mathrm{dL}$, international normalized ratio or prothrombin time or international normalized thromboplastin time $\leq 1.5$ times upper limit of normal). Premenopausal women were required to have a negative pregnancy test, and all participants of childbearing potential were required to use contraception. Patients may have received any number of prior lines of therapy, but could not have received prior therapy with anti-PD-(L)1 ICIs. Patients with oncogene-addicted or hormone-responsive tumors who were receiving either targeted therapy or hormonal therapy prior to study enrollment were permitted to continue this therapy concurrently with pembrolizumab, determined on a case-by-case basis, if there was published and acceptable safety data of the combination in question. Patients were permitted to enroll if they were receiving corticosteroids, as long as the steroid requirement was non-escalating. Patients who underwent major surgery to the brain within 3 weeks, had concurrent brain metastasis with mass effect, or a history of steroid-requiring pneumonitis were not eligible. Prior radiation therapy (RT) to LMM was allowed, if administered more than 3 months before study start or to non-target areas of LMM. An area of LMM that had received RT at any time could not be used as a target lesion for the assessment of CNS response.

\section{Study design}

This was an investigator-initiated, open-label singleinstitution phase II trial of pembrolizumab monotherapy in patients with LMM from any solid tumor. Pembrolizumab was administered intravenously at a flat dose of $200 \mathrm{mg}$ every 3 weeks until disease progression or unacceptable toxicity. Screening investigations were completed within 2 weeks of study start and included standard laboratory investigations, CSF sampling for cytology, and a pretreatment gadolinium-enhanced MRI scan of the brain and total spine. During the trial, patients were monitored with a clinical visit, adverse event (AE) assessment, and routine laboratory tests at the time of each dose of pembrolizumab. Patients had blood draws, CT imaging of the body, and a contrast-enhanced MRI scan of the brain and total spine after 2 and 4 cycles of pembrolizumab, and every 6-9 weeks thereafter until disease progression or coming off study. Patients were followed for 90 days after the end of therapy for $\mathrm{AE}$ monitoring or 30 days after the end of therapy if a new anti-cancer therapy was initiated. Serial CSF samples were obtained by lumbar puncture and assessed for cytopathology, genomic and immunologic assays pre-treatment, and after 2 and 4 cycles of pembrolizumab. We assayed CSF for the presence of t-DNA by chromosomal copy number changes (aneuploidy) using a PCR-based approach (RealSeqS). We assessed CSF for immunologic features by 16-color flow cytometry, immunophenotyping, and multiplex cytokine panels, as outlined later.

\section{Study endpoints}

The primary endpoint of the study was CNS response assessed after 12 weeks of therapy using prospectively defined criteria. ${ }^{7} \mathrm{CNS}$ response was defined either as radiologic, cytologic, or clinical response to therapy assessed by the treating investigator, and outlined in the protocol (online supplemental table 1). Radiologic response was defined as a reduction in size of LMM on gadoliniumenhanced MRI of the brain and total spine including T1-weighted, T2-weighted, fluid-attenuated inversion recovery, and post-gadolinium sequences. All radiologic responses were confirmed by a dedicated study neuroradiologist. Cytologic response was defined as conversion of positive to negative CSF cytology on two consecutive CSF samples taken at the time of study treatment. Clinical response was defined as an improvement in objective neurologic signs or symptoms attributable to LMM by the treating investigator. Retrospective analysis using LM-RANO criteria $^{11}$ was also performed for all patients who were assessed for response by the study radiologist (NA). Secondary endpoints included CNS-PFS, OS and 
safety. Adverse events were graded according to Common Terminology Criteria for Adverse Events (CTCAE version 4.0). OS was defined as time from pembrolizumab start until death from any cause. CNS-PFS was defined as time from pembrolizumab start until radiologic, cytologic or clinical progression in LMM, whichever came first.

\section{Correlative analysis}

RealSeqS can sensitively detect aneuploidy using a single PCR primer pair. In RealSeqS, for each sample a single primer pair was used to amplify $\sim 350,000$ loci spread throughout the genome. ${ }^{12}$ One of the primers included a unique identifier sequence (UID) as a molecular barcode of 16 degenerate bases to reduce error rates associated with PCR and sequencing. Massively parallel sequencing was performed on an Illumina HiSeq 4000. Sequencing data were processed to identify single chromosomal arm gains or losses using the Within-Sample AneupLoidy Detection (WALDO), incorporated into the RealSeqS work flow. ${ }^{13}$ WALDO used a set of normal non-cancer healthy plasma controls as references (that were excluded from all other analyses), and based on the closest reference generated a Z score, for likelihood of alterations on each acrocentric chromosomal arm. WALDO further incorporated a support vector machine (SVM) to discriminate between aneuploid and euploid samples and to provide a summary Genome Aneuploid Score statistic. The SVM classifier was trained using 1334 normal euploid plasma samples and 2651 aneuploid samples. The software and all datasets used to generate the scores for this manuscript have been posted and are available online (https://doi.org/10. 5281/zenodo.3656943). A cut-off of 0.333 was selected for a positive call; this cut-off was selected based on specificity of 98\% reported in the manuscript on 1348 normal samples. ${ }^{13}$

The immunologic features of collected CSF samples were analyzed by flow cytometry following immunostaining for CD3 to identify T cells (anti-CD3-Percp Cy5.5, 1:50, clone: OKT3, Cat\#45-0037-42; Invitrogen) and CD19 to identify B cells (anti-CD19-FITC, 1:50, clone: HIB19, Cat\#11-019942; Invitrogen). Cells were incubated with antibodies on ice for 30 minutes in the dark, followed by washing, re-suspension, and analysis via MACSQuant Analyzer 10 (Miltenyi Biotec). Expression profiles of each cell type was analyzed via FlowJo_V10 (Tree Star Inc., Ashland, OR) software. T cells and B cells were enumerated for all baseline and on-treatment samples based on flow cytometry panels. The V-PLEX Plus Human Cytokine 30-Plex assay was used per manufacturer's instructions (Meso Scale Diagnostics, CA) to characterize cytokine profiles. Cytokines and chemokines were annotated on baseline and on-treatment samples and represented by heatmaps, using absolute and rank order values, excluding a coefficient of variation in excess of $10 \%$.

\section{Statistical analysis}

All enrolled patients who received at least one dose of study therapy were included in this analysis. CNS response proportion and its associated CI was calculated using Clopper-Pearson exact method, and the study size

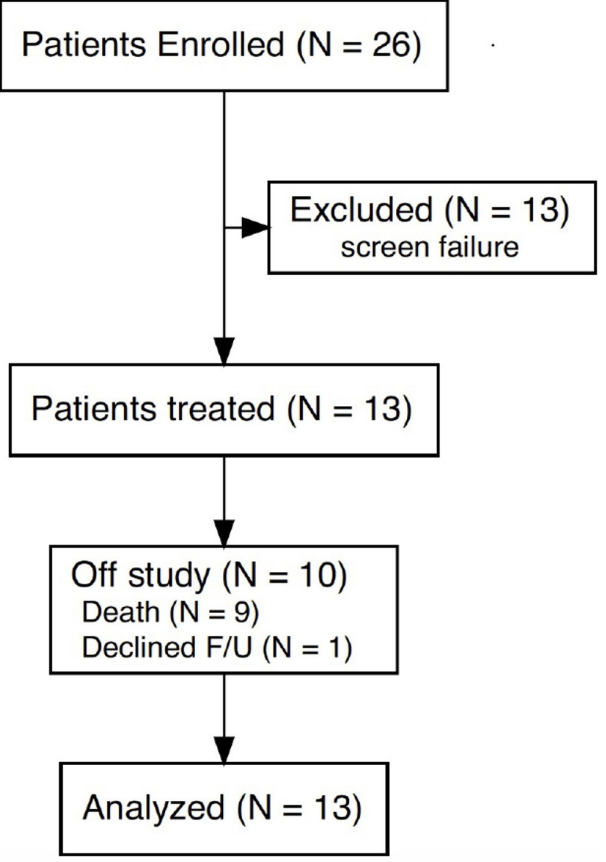

Figure 1 Consort diagram.

was determined as 16 analyzable patients to control the estimation precision prospectively (appendix I). KaplanMeier method was used to estimate PFS and OS and the log-rank test was used to compare differences in CNSPFS and OS rates by baseline cytologic LMM. Treatmentrelated adverse events (TRAEs) were reported for all patients. Baseline and on-treatment genomic and immunologic data from CSF samples were presented descriptively, and correlated with CNS response and OS.

\section{RESULTS}

Between February 15, 2017 and December 9, 2019, 26 patients were screened for study entry. The study was stopped early for poor accrual. Thirteen patients received at least one dose of pembrolizumab, and were included in this analysis (figure 1).

\section{Baseline characteristics}

Patient and disease characteristics at baseline are listed in table 1 . The median age was 57 years (range: 22-79). The majority of patients $(n=8,62 \%)$ had tumors not traditionally responsive to ICI monotherapy ("ICI nonresponsive": breast carcinoma $=5,39 \%$; high-grade glioma $=3,23 \%$ ), while $38 \%$ had tumors that traditionally respond to ICI monotherapy (ICI-responsive $=5$, $38 \%)$, consisting of NSCLC $(n=3,23 \%)$, head and neck carcinoma (HNSCC) $(\mathrm{n}=1,8 \%)$, and cutaneous squamous carcinoma of the skin (CSC) $(n=1,8 \%)$. Of the patients with breast carcinoma, three had HR-positive/ HER2-positive breast cancers, and two had HR-positive/ HER-2-negative tumors. One patient with HR-positive/ HER2-positive breast cancer continued trastuzumab and tamoxifen during the study concurrently with pembrolizumab, based on safety data of this combination that 
Table 1 Baseline patient demographics and disease characteristics

\begin{tabular}{ll}
\hline Patient demographics & Total $(\mathbf{n}=\mathbf{1 3}, \mathbf{\%})$ \\
\hline Median age, years (range) & $57(22-79)$ \\
Sex & \\
\hline Female & $7(53.8)$ \\
\hline Male & $6(46.2)$ \\
\hline Race & \\
\hline Caucasian & $9(69.2)$ \\
Asian & $2(15.4)$ \\
Unknown & $2(15.4)$ \\
\hline ECOG Performance Status & \\
\hline 0 & $12(92.3)$ \\
\hline 1 & $1(7.7)$
\end{tabular}

Primary Tumor Diagnosis

$\begin{array}{ll}\text { Non-small cell Lung Cancer } & 3(23.1) \\ \text { Head and Neck Squamous Carcinoma } & 1(7.6) \\ \text { Cutaneous Squamous Carcinoma of Skin } & 1(7.6) \\ \text { Breast carcinoma† } & 5(38.4) \\ \text { High-grade Glioma } & 3(23.1)\end{array}$

Presence of Extracranial Disease

$\begin{array}{ll}\text { Yes } & 4(30.7) \\ \text { No } & 9(69.3) \\ \text { Neurologic Symptoms of LMM } & \\ \text { Yes } & 13(100) \\ \text { No } & 0(0)\end{array}$

Baseline CSF cytology

\begin{tabular}{ll}
\hline Positive & $6(46.2)$ \\
\hline Negative & $7(53.8)$ \\
\hline Median CSF White Cell Count (range) & $6(1-131)$ \\
\hline $\begin{array}{l}\text { Median Serum Absolute Lymphocyte count (range) } \\
\text { Disease Characteristics }\end{array}$ & $0.91(0.3-2.4)$ \\
\hline Prior Chemotherapy & \\
\hline Yes & $10(76.9)$ \\
\hline No & $3(23.1)$ \\
\hline Prior Brain/Spine Radiotherapy & $10(76.9)$ \\
\hline Yesł & $3(23.1)$ \\
\hline No & \\
\hline No. of Prior Lines of Systemic Therapy & $3(23.1)$ \\
\hline 0 & $1(7.7)$ \\
\hline 1 & $9(69.2)$ \\
\hline $2+$ & \\
\hline Any corticosteroids at the time of study enrollment & \\
\hline Yes & $5(38.4)$ \\
\hline No & $8(61.5)$ \\
\hline
\end{tabular}

*Two NSCLCs were PD-L1 <1\%, 1 harbored a MET-exon14 skipping alteration; 1 had no oncogenic driver mutation; 1 NSCLC harbored an NRAS mutation and was PD-L1 30\%.

†3 patients had HER2-positive breast cancers; and 2 patients had ERpositive/PR-negative/HER-2-negative breast cancers.

$\ddagger$ Whole-Brain Radiation Therapy $=4$; Stereotactic Radiosurgery $=1$; Focal RT to $L M M=5$; all received $>3$ months before study enrollment.

ECOG, Eastern Cooperative Oncology Group. had been presented prior to enrollment. ${ }^{14}$ No patients received targeted therapy concurrently with protocoldirected pembrolizumab in this study. Of the patients with NSCLC ( $\mathrm{n}=3$ ), two patient's tumors were PD-L1-negative $(<1 \%)$, one of which harbored a MET-exon 14 skipping alteration, while the other did not harbor an oncogenicdriver alteration. The third NSCLC tumor had a PD-L1 score of 30\% (22C3) and harbored an NRAS Q61K mutation. The majority of patients in this study had LMM only without disease in the body $(n=11,84.6 \%)$. Interestingly, in slightly majority of enrolled patients, cytologic LMM was not detectable using CSF cytopathology ( $\mathrm{n}=7,53.8 \%)$. Overall, most of the study population was heavily pretreated $(2+$ prior lines of systemic therapy $=9,69.2 \%)$ and had received prior radiation to the brain $>3$ months prior to study enrollment $(\mathrm{n}=10,76.9 \%)$. A minority of patients received non-escalating corticosteroid therapy at the time of enrollment $(n=5,38 \%)$, the dose at enrollment was equivalent to prednisone $25 \mathrm{mg}$ /day in one patient, while all others were equivalent to prednisone $\leq 10 \mathrm{mg}$ /day for symptomatic management of LMM.

\section{Efficacy}

Overall CNS response among the 13 patients treated with pembrolizumab was assessed (table 2). Six patients received more than four doses of pembrolizumab $(\mathrm{n}=6 / 13,46 \%)$. CNS response was assessed using predefined study criteria as outlined in the protocol or retrospectively using LM-RANO criteria, as outlined in table 2. Since LM-RANO criteria do not differentiate between partial and complete responses to treatment and in this manuscript CNS response included stable disease, the CNS response rate from both assessments of response were identical. CNS response was observed in $38 \%(\mathrm{n}=5 / 13,95 \% \mathrm{CI} 13.9 \%$ to $68.4 \%)$, while most had progressive disease (PD) in the CNS ( $\mathrm{n}=8,61.5 \%)$. Two patients achieved complete responses (CR) in the CNS after pembrolizumab, one had a partial response $(7.7 \%)$, and two $(15.4 \%)$ had stable disease. Both patients with CRs had ICI-responsive tumors (CSC; METexon14+NSCLC) (figure 2). The patient with NSCLC had received prior crizotinib and sustained prolonged stable disease from this therapy, as well as a short-lived response to second-line chemotherapy. This patient received five doses of study therapy and had complete radiographic resolution of LMM, but developed grade 2 pneumonitis and grade 2 inflammatory arthritis, necessitating treatment hold. During this period, the patient had PD in the body and subsequently died, while CNS disease did not recur (OS 9 months). Interestingly, this patient commenced study therapy while receiving corticosteroids at a dose of prednisone $\leq 10 \mathrm{mg}$ /day. The other patient with a CNS CR had newly diagnosed CSC and received 17 doses of study therapy as first-line treatment, with ongoing CNS CR and OS 3+ years from ICI start. Both patients who sustained CRs to study therapy had not received prior radiation therapy to LMM. The duration of response to pembrolizumab by CNS radiologic response 
Table 2 Response to treatment

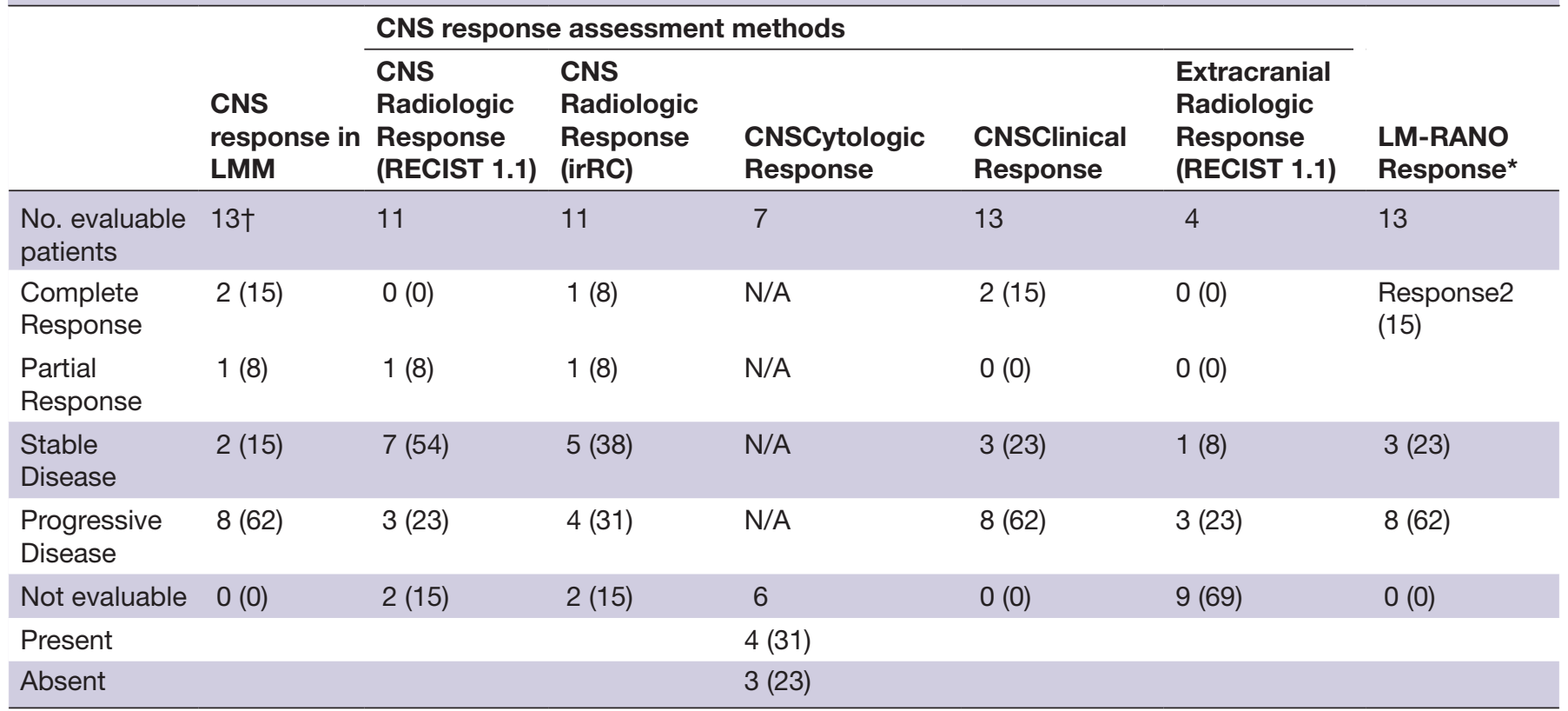

*LM-RANO: Leptomeningeal Metastasis Response Assessment in Neuro-Oncology.

†Primary Response assessment for Overall CNS response as radiologic in 10 cases, and clinical in 3 cases.

CNS, central nervous system; irRC, immune-related response criteria; LMM, leptomeningeal metastasis; N/A, not applicable; RECIST, response evaluation criteria for solid tumors.

and tumor type is depicted in figure 3. The median CNSPFS was 2.9 months (95\% CI 1.3 to NR) and the median OS was 4.9 months (95\% CI 3.7 to NR) (figure 4). While not statistically significant, the presence of cytologic LMM was associated with numerically poorer OS versus those without cytologically detectable LMM (3.7 months vs 10.3 months, log-rank $\mathrm{p}=0.29)$ (online supplemental figure 1$)$.

\section{Adverse events}

TRAEs occurred in $38.5 \%$ of patients $(n=5 / 13)$, and the majority were low grade $(23.1 \%, 3 / 13)$ (online supplemental table 2). There were no high-grade immunerelated adverse events (irAEs). Drug therapy was withheld for TRAEs that were also irAEs in both patients with CNS CR (NSCLC: pneumonitis; CSC: pain in extremity, joint reduced range of motion).

\section{CSF analysis}

CSF was obtained for cytopathology, t-DNA, and immunologic analysis (flow cytometry, immunophenotyping, cytokine analysis) at baseline in all patients $(n=13)$, and in patients with available samples after two $(n=10)$ and four cycles $(n=4)$ of pembrolizumab.

Seven patients had positive CSF cytopathology (53.8\%) at baseline. No CSF samples had significant contamination from peripheral blood (red blood cells $<500 / \mu \mathrm{L}$ ) for all patients. Positive CSF cytopathology did not correlate with CSF white blood cell count, protein, glucose, or baseline radiographic LMM. There were no significant differences in CNS response or OS $(\mathrm{p}=0.278)$ in patients with positive versus negative baseline CSF cytopathology (online supplemental figure 1). Given the limitations of traditional CSF cytopathology, we evaluated the sensitivity of CSF t-DNA by aneuploidy assay, for the diagnosis of LMM and associations with CNS response and OS. The sensitivity of CSF for the detection of LMM by t-DNA aneuploidy was $84.6 \%$ (95\% CI $54.6 \%$ to $98.1 \%$ ) and $53.9 \%$ (95\% CI $25.1 \%$ to $80.8 \%$ ) by cytopathology (online supplemental table 3 ). We were unable to assess specificity as all patients in the cohort had LMM. There were no significant associations between CSF t-DNA by aneuploidy assay and either CNS response $(\mathrm{p}=0.65)$ or OS $(0.79)$.

Flow cytometric analysis demonstrated a median of 35024 live cells (IQR 14,043, 117,736) per sample. T cells $\left(\mathrm{CD}^{+}\right.$expression) comprised $44 \%$ (SD 36\%) of the total, while B cells $\left(\mathrm{CD} 19^{+}\right.$expression) comprised a smaller proportion (mean: 3.9\%; SD 7.7\%). Baseline CSF cytokine analysis demonstrated that both patients who sustained CNS CRs (\#3, \#11) as well as two patients with short-lived responses (\#8, \#24) clustered together, while patients with clear CNS PD (\#1, \#4, \#10, \#16) clustered differently (online supplemental figure 2). Lower levels of baseline pro-inflammatory cytokines were seen in patients 3,11 , 8 , and 24 . When examining the log-fold change of cytokines comparing baseline with the post cycle 2 visit, once again both CRs clustered together (online supplemental figure 2), exhibiting a further reduction in the levels of pro-inflammatory cytokines.

\section{DISCUSSION}

In this prospective clinical trial, we identified that $38 \%$ of patients with LMM from solid tumors had CNS responses to pembrolizumab. The median CNS-PFS and OS was 
A

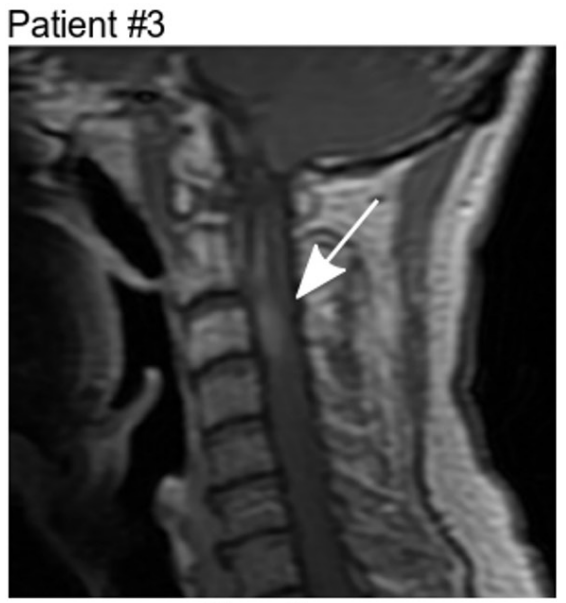

Baseline

\section{B}

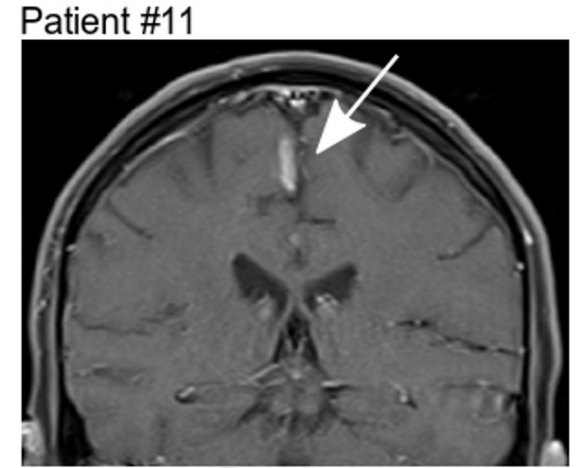

Baseline

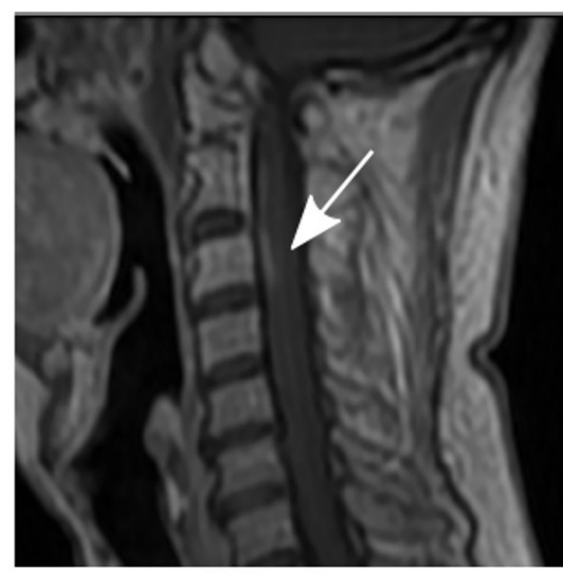

2 Months

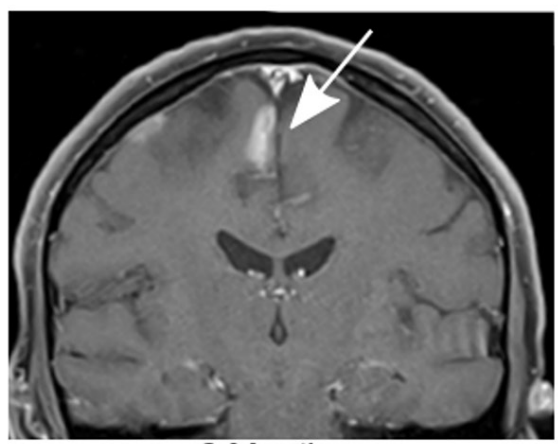

2 Months

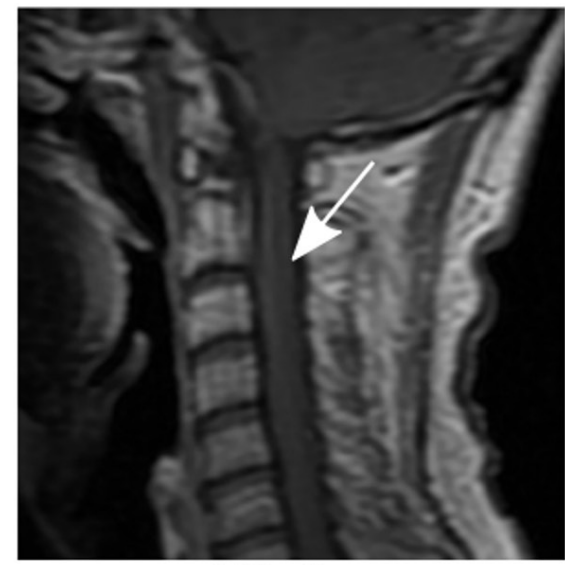

4 Months

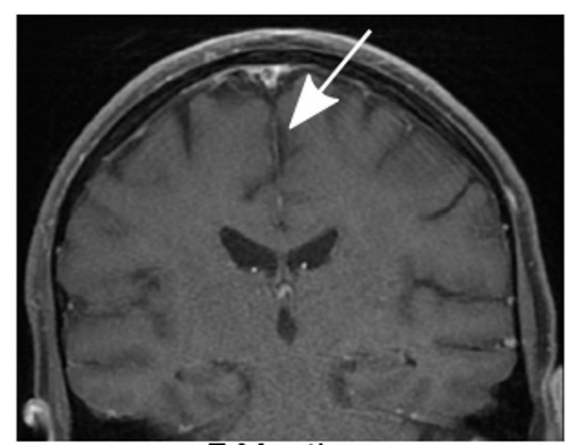

7 Months

Figure 2 Radiologic imaging in complete central nervous system responders to pembrolizumab.

2.9 months and 4.9 months, respectively, in keeping with published prospective studies in LMM. ${ }^{715-17}$ Pembrolizumab was also safe to administer, with a low incidence of high-grade TRAEs. Interestingly, two patients with ICIresponsive tumors (NSCLC, CSC) demonstrated complete responses in the CNS, clinically and radiographically.

The main goal of this investigator-initiated trial was to identify whether anti-PD-1 could lead to CNS responses. We adopted stringent criteria for inclusion and assessment of LMM, only enrolling patients with clearly measurable radiographic disease or cytologic proof of LMM, followed by multidisciplinary consensus. While this led to a high rate of screen failure, it also ensured reliable assessment of CNS response. OS has been used as a primary endpoint for several studies in LMM, but may not account for prolonged OS seen in subsets of patients with oncogeneaddicted cancers and LMM. OS was assessed as secondary endpoint, together with CNS-PFS and safety. We included patients in our study who had received prior RT to the CNS, but restricted this to 3+ months before ICI start, to limit the effect on CNS response assessment. Patients receiving non-escalating doses of corticosteroids were also allowed to enroll despite the potential deleterious effects on ICI response, ${ }^{18}$ and only one patient received a dose higher than prednisone $10 \mathrm{mg}$ / day, which has historically been deemed acceptable in ICI studies.

The study population in this trial was heavily pretreated, comprised mainly of patients with tumors that are not typically responsive to ICI monotherapy, and with CNS-only disease. This was unsurprising since anti-PD-1 was already available for many cancers, and our study did not require measurable disease in the body. Despite this, $38 \%$ of enrolled patients exhibited CNS responses from pembrolizumab. Re-analysis of responses using the recently published consensus LM-RANO criteria matched our protocol-defined criteria for assessing response based on neurological examination, radiographic features, and CSF cytopathology. Objective responses occurred in patients with tumor types that typically respond to antiPD-1 (CR: NSCLC, CSC; PR: NSCLC). Interestingly, two patients sustained CRs in their LMM, substantiating that certain patients may derive prolonged benefit. A potential explanation for these CNS responses is that activated T cells were able to reach the CNS through a less effective blood-brain barrier, owing to the presence of LMM. One other prospective study has explored the use of anti-PD-1 in patients with LMM. This study enrolled 18 patients with breast cancer, with the goal of assessing OS rate from 


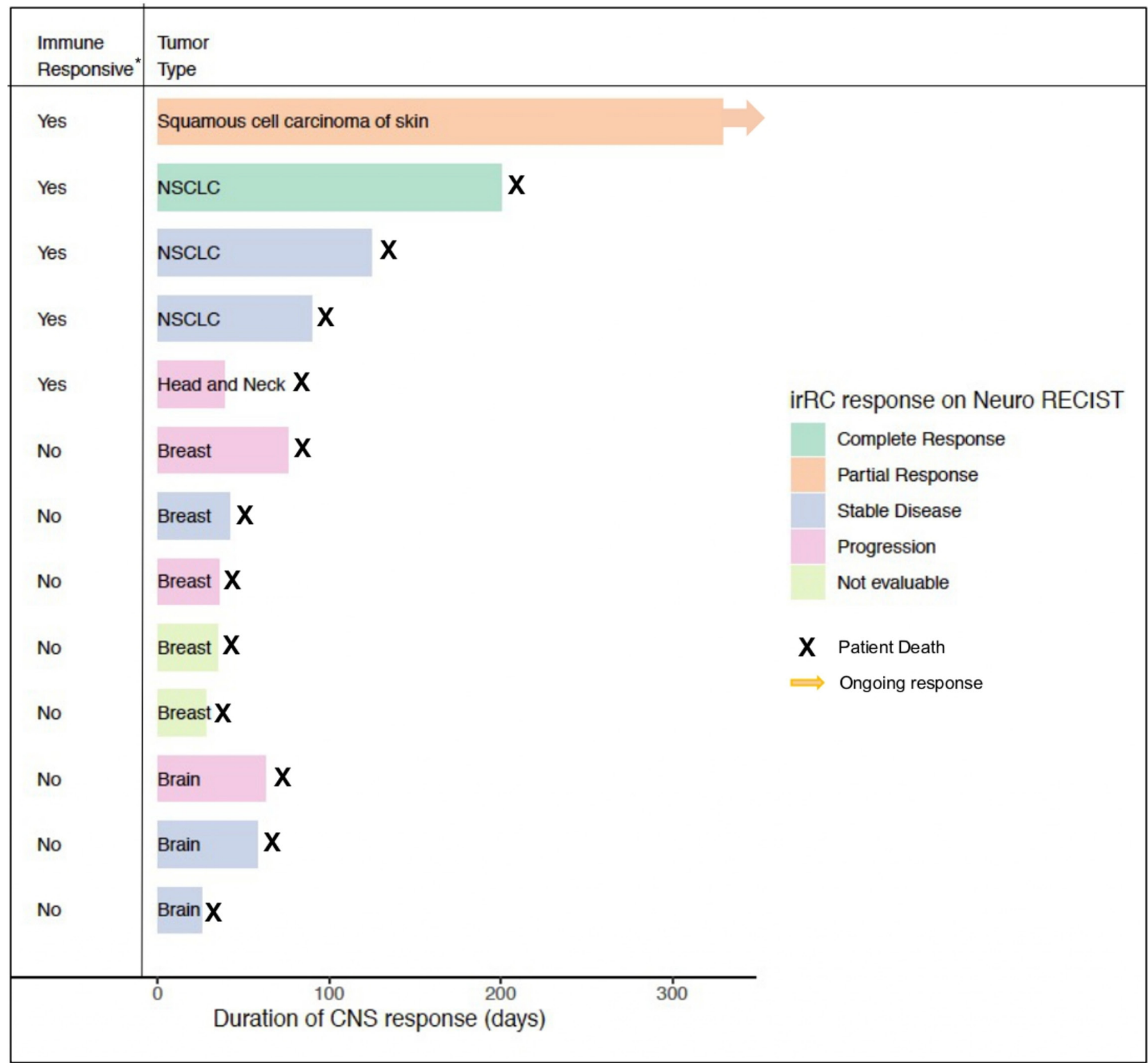

Figure 3 Swimmer plot of radiologic response duration for patients with leptomeningeal metastasis treated with pembrolizumab. CNS, central nervous system; NSCLC, non-small cell lung cancer.

pembrolizumab at 3 months. Consistent with our survival outcomes, this study and others in similar cohorts ${ }^{19-21}$ demonstrated an OS rate of $60 \%$ at 3 months.

Uniquely, in our study we aimed to elucidate the biologic features of CNS response to pembrolizumab. We undertook a comprehensive genomic and immunologic assessment of the CSF from enrolled patients at baseline and serially on-treatment. We observed that CSF t-DNA by aneuploidy assay may be sensitive for detection of LMM, and that there may be important immunologic subsets of patients relative to ICI response based on multiplex cytokine assays. Lower pro-inflammatory cytokine levels have been correlated in prior studies to lower tumor burden in the CNS. ${ }^{22}{ }^{23}$ Thus, this may account for lower
CSF cytokine levels in selected responders in our cohort and further reductions in those with a declining burden of disease (response to therapy). Given the small sample size, our findings are exploratory in nature. Based on these interesting results, studies examining CSF t-DNA in larger numbers of patients with suspected LMM are underway to assess the potential diagnostic utility of this assay, as well as further immunologic studies on those with differing ICI responses.

Lastly, we identified that pembrolizumab was well tolerated in this population of patients, who are often deemed too unwell to pursue systemic therapy. These data have important implications for treatment choices in patients with LMM since other treatments for LMM are 


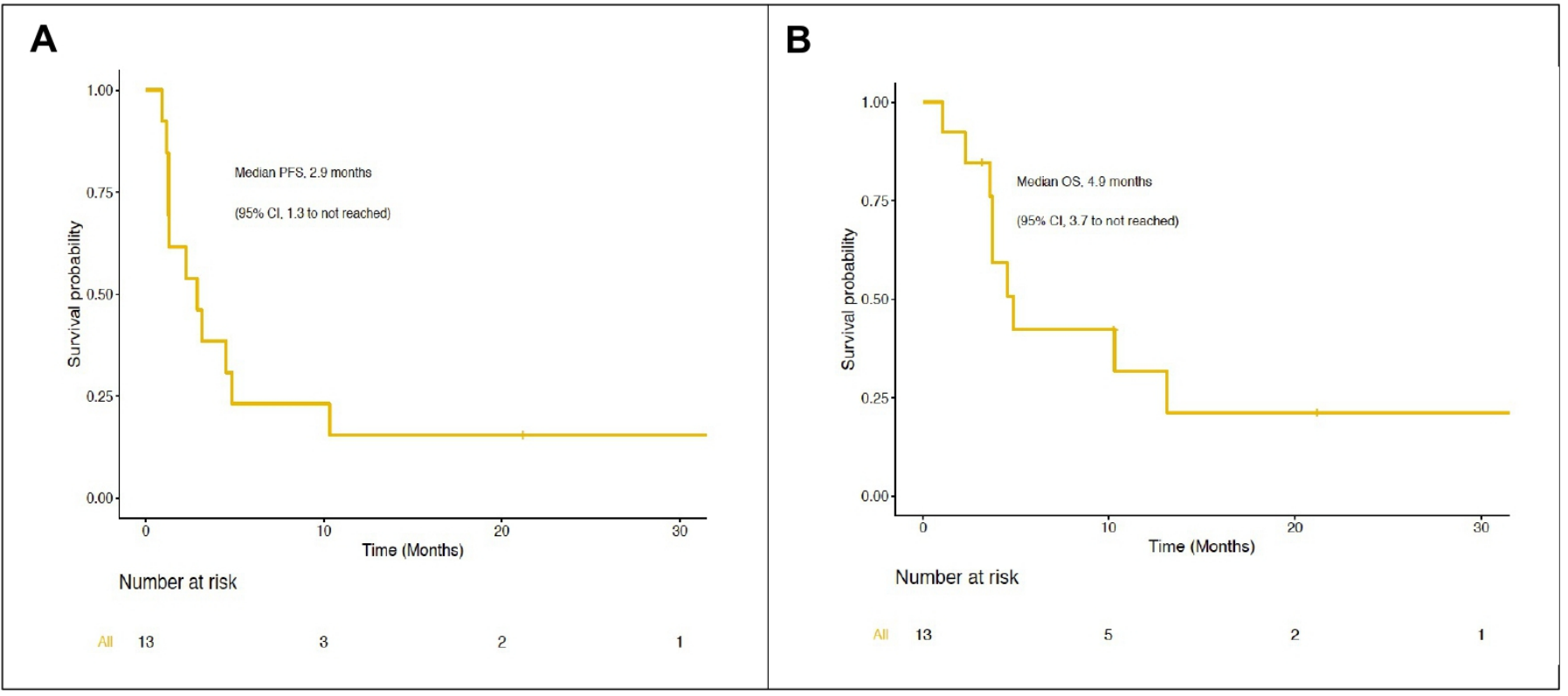

Figure 4 Central nervous system progression-free survival and overall survival for patients with leptomeningeal metastasis treated with pembrolizumab.

associated with much higher rates of toxicity,and in-patient administration.

While our study demonstrated several important findings, there were also limitations. We only enrolled physically robust patients who could tolerate clinical trial treatment. Thus, our study may not represent the entire population of patients with LMM, who tend to have a poor ECOG PS. Given the rarity of LMM and strict eligibility criteria, our study closed early for poor accrual. We were thus unable to estimate CNS response with the same planned precision. Despite this, we identified compelling CNS response, survival, and safety findings. In order to expand the reach of this study, we chose to enroll patients with any solid tumor. While this may have broadened the applicability of our findings, including patients with tumors not traditionally responsive to ICIs may have resulted in a lower CNS response rate. In terms of assessment of CNS response, when this study was developed there was no consensus regarding optimal radiographic response assessment of LMM. We thus used published but older CNS response criteria for LMM that incorporated radiographic, cytologic, and clinical response, which may be less applicable using modern MRI techniques, and should be considered in the interpretation of CNS response in this study. ${ }^{7}$ Subsequently published LM-RANO criteria assess response using similar criteria (MRI, CSF cytology, neurologic examination) ${ }^{11}$ to our study; however, there is controversy over its reproducibility. ${ }^{24}$ While findings were not statistically significant, we also note relatively disparate outcomes for patients in this study based on the presence or absence of detectable CSF cytology. In addition, while CNS response is a clinically relevant endpoint for patients with LMM, given the challenges in assessing LMM response, OS (or 3-month landmark OS) is now more commonly used as an endpoint for trials of novel agents in LMM. Lastly, while we pursued investigation of potential CSF biomarkers, the number of immune cells present in the CSF and sample size of our study limited the ability to reach statistically significant conclusions or in analyzing serial samples over time. Future CSF work using single-cell approaches ${ }^{25} 26$ and multicenter collaborations should be sought.

In conclusion, in this phase II trial of pembrolizumab for patients with LMM from solid tumors, $38 \%$ of patients demonstrated CNS responses by predefined study criteria. Pembrolizumab was well tolerated, and survival outcomes were consistent with published studies. Novel CSF biomarker studies in enrolled patients demonstrated that CSF aneuploidy of t-DNA may be sensitive for the detection of LMM, and that immunologic subsets of ICIresponse based on cytokine profiles warrant further study. These data support further study of ICIs in patients with LMM in ICI-responsive tumor types and an adequate performance status.

\section{Author affiliations}

${ }^{1}$ Department of Oncology, Sidney Kimmel Comprehensive Cancer Center, Johns Hopkins University, Baltimore, Maryland, USA

${ }^{2}$ Department of Immunology, The Bloomberg-Kimmel Institute for Cancer Immunotherapy, Johns Hopkins University, Baltimore, Maryland, USA

${ }^{3}$ Department of Oncology, Beaumont Hospital and RCSI University of Health Sciences, Dublin, Ireland

${ }^{4}$ Department of Neurology, John Hopkins Medicine, Johns Hopkins University, Baltimore, Maryland, USA

${ }^{5}$ Department of Neurosurgery, Johns Hopkins University, Baltimore, Maryland, USA ${ }^{6}$ Department of Biostatistics, Sidney Kimmel Comprehensive Cancer Center, John Hopkins University, Baltimore, Maryland, USA

${ }^{7}$ Cancer Research@UCC, College of Medicine and Health, University College Cork, Cork, Ireland

${ }^{8}$ Department of Immunology, Johns Hopkins Medicine Sidney Kimmel Comprehensive Cancer Center, Baltimore, Maryland, USA

${ }^{9}$ Ludwig Center for Cancer Genetics and Therapeutics, Johns Hopkins University, Baltimore, Maryland, USA 
${ }^{10}$ Sol Goldman Pancreatic Cancer Research Center, Johns Hopkins University, Baltimore, Maryland, USA

${ }^{11}$ Department of Radiation Oncology, Sidney Kimmel Comprehensive Cancer. John Hopkins University, Baltimore, Maryland, USA

${ }^{12}$ Division of Radiology, Johns Hopkins Hospital, Baltimore, Maryland, USA

Twitter Jarushka Naidoo @DrJNaidoo and Joanne Riemer @jriemer3

Acknowledgements We thank L. Rajbhandari for technical assistance and B. Vogelstein for his input into the CSF t-DNA aneuploidy assay.

Contributors Study conception and design: JN, KCS, WF, CH, AC-G, PMF, NA, CB, AV, KJR, LK, JRB, SAG. Patient accrual and management on study: JN, KCS, RC, CAS-M, EJL, MH, PMF, JR, AB, KJR, LK, BP, JRB, SAG. Study data analysis: JN, KCS, WF, $\mathrm{CH}, \mathrm{CD}, \mathrm{AB}, \mathrm{KWK}, \mathrm{NP}, \mathrm{CB}, \mathrm{AV}, \mathrm{JRB}, \mathrm{SAG}$. Manuscript preparation and writing: JN, KCS, WF, CH, AC-G, RC, CAS-M, EJL, MH, PMF, CD, JR, AB, KJR, LK, BP, NA, KWK, NP, $\mathrm{CB}, \mathrm{AV}$, JRB, SAG. Manuscript approval: JN, KCS, WF, CH, AC-G, RC, CAS-M, EJL, MH, $P M F, C D, J R, A B, K J R, L K, B P, N A, K W K, N P, C B, A V, J R B, S A G$.

Funding This work was supported by an investigator-initiated trial research grant from Merck (Naidoo); and NIH RA37CA230400, U01CA230691 (Bettegowda, Vogelstein).

Competing interests JN: research funding: Merck, AstraZeneca; consulting: AstraZeneca, Bristol-Myers Squibb; Takeda, Daiichi Sankyo, Roche/Genentech; Honoraria: Merck, AstraZeneca, Bristol-Myers Squibb, Roche/Genentech. CD: consultant: Depuy-Synthes, Bionaut Labs; royalties: Thrive (through Johns Hopkins University). $\mathrm{CH}$ : research funding: $\mathrm{NCI} / \mathrm{NIH}$; consultant: Merck \& $\mathrm{Co}$. CAS-M: research funding: NCI/NIH, Pfizer, AstraZeneca, Novartis, BMS, Tesaro/ GSK; consultant: BMS, Seattle Genetics, Genomic Health, Athenex, Polyphor, Halozyme. MH: consultant: BTG International, NewLink Genetics, Celgene, AbbVie. PMF: consultant: AstraZeneca, Abbvie, Amgen, BMS, Janssen; research funding: AstraZeneca, BMS. CD, KWK, NP, CB, and BP are founders of, hold equity in, and are consultants to Thrive and Personal Genome Diagnostics. KWK and NP are on the Board of Directors of Thrive. KWK is a consultant to Sysmex, Eisai, and CAGE Pharma. KWK and NP are consultants to Neophore. CB is a consultant to DepuySynthes and Bionaut Labs. CD is a consultant to Thrive and is compensated with income and equity. The companies named above as well as other companies have licensed previously described technologies related to the work described in this paper from Johns Hopkins University. CD, CB, KWK, and NP are inventors on some of these technologies. Licenses to these technologies are or will be associated with equity or royalty payments to the inventors as well as to Johns Hopkins University. The terms of all of these arrangements are being managed by Johns Hopkins University in accordance with its conflict of interest policies. KJR: research funding: Accuray, Elekta $A B$; travel expenses: Brainlab, Accuray, Elekta AB; honoraria: Elekta $A B$; data safety monitoring board: BioMimetixRC; research funding to institution from Novartis, Genentech, Merck, Macrogenics, Puma Biotechnology. Unrestricted educational grant from Pfizer.

Patient consent for publication Not required.

Ethics approval This study was approved by the Johns Hopkins University Institutional Review Board (IRB), under the protocol number J16156.

Provenance and peer review Not commissioned; externally peer reviewed.

Data availability statement Data are available on reasonable request.

Supplemental material This content has been supplied by the author(s). It has not been vetted by BMJ Publishing Group Limited (BMJ) and may not have been peer-reviewed. Any opinions or recommendations discussed are solely those of the author(s) and are not endorsed by BMJ. BMJ disclaims all liability and responsibility arising from any reliance placed on the content. Where the content includes any translated material, BMJ does not warrant the accuracy and reliability of the translations (including but not limited to local regulations, clinical guidelines, terminology, drug names and drug dosages), and is not responsible for any error and/or omissions arising from translation and adaptation or otherwise.

Open access This is an open access article distributed in accordance with the Creative Commons Attribution Non Commercial (CC BY-NC 4.0) license, which permits others to distribute, remix, adapt, build upon this work non-commercially, and license their derivative works on different terms, provided the original work is properly cited, appropriate credit is given, any changes made indicated, and the use is non-commercial. See http://creativecommons.org/licenses/by-nc/4.0/.

\section{ORCID iDs}

Jarushka Naidoo http://orcid.org/0000-0002-3470-8686
Evan J Lipson http://orcid.org/0000-0003-2976-0911

Matthias Holdhoff http://orcid.org/0000-0002-3285-3484

\section{REFERENCES}

1 Prins RM, Vo DD, Khan-Faroogi $\mathrm{H}$, et al. NK and CD4 cells collaborate to protect against melanoma tumor formation in the brain. J Immunol 2006;177:8448-55.

2 Wilson EH, Weninger W, Hunter CA. Trafficking of immune cells in the central nervous system. J Clin Invest 2010;120:1368-79.

3 Margolin K, Ernstoff MS, Hamid O, et al. Ipilimumab in patients with melanoma and brain metastases: an open-label, phase 2 trial. Lancet Oncol 2012;13:459-65.

4 Long GV, Atkinson V, Lo S, et al. Combination nivolumab and ipilimumab or nivolumab alone in melanoma brain metastases: a multicentre randomised phase 2 study. Lancet Oncol 2018;19:672-81.

5 Goldberg SB, Schalper KA, Gettinger SN, et al. Pembrolizumab for management of patients with NSCLC and brain metastases: longterm results and biomarker analysis from a non-randomised, openlabel, phase 2 trial. Lancet Oncol 2020;21:655-63.

6 Long GV, Atkinson VG, Lo S, et al. Long-term outcomes from the randomized phase II study of nivolumab (nivo) or nivo+ipilimumab (IPI) in patients (PTS) with melanoma brain metastases (Mets): antiPD1 brain collaboration (ABC). Ann Oncol 2019;30:v534.

7 Grossman SA, Finkelstein DM, Ruckdeschel JC, et al. Randomized prospective comparison of intraventricular methotrexate and thiotepa in patients with previously untreated neoplastic meningitis. Eastern Cooperative Oncology Group. J Clin Oncol 1993;11:561-9.

8 Glantz MJ, Jaeckle KA, Chamberlain MC, et al. A randomized controlled trial comparing intrathecal sustained-release cytarabine (DepoCyt) to intrathecal methotrexate in patients with neoplastic meningitis from solid tumors. Clin Cancer Res 1999;5:3394-402.

9 Ahn M-J, Chiu C-H, Cheng Y, et al. Osimertinib for patients with leptomeningeal metastases associated with EGFR T790M-positive advanced NSCLC: the aura leptomeningeal metastases analysis. $J$ Thorac Oncol 2020;15:637-48.

10 Brastianos PK, Lee EQ, Cohen JV, et al. Single-arm, open-labe phase 2 trial of pembrolizumab in patients with leptomeningeal carcinomatosis. Nat Med 2020;26:1280-4.

11 Chamberlain M, Junck L, Brandsma D, et al. Leptomeningeal metastases: a RANO proposal for response criteria. Neuro Oncol 2017:19:484-92.

12 Douville C, Cohen JD, Ptak J, et al. Assessing aneuploidy with repetitive element sequencing. Proc Natl Acad Sci U S A 2020;117:4858-63.

13 Douville C, Springer S, Kinde I, et al. Detection of aneuploidy in patients with cancer through amplification of long interspersed nucleotide elements (LINEs). Proc Natl Acad Sci U S A 2018;115:1871-6.

14 Loi S, Giobbie-Hurder A, Gombos A, et al. Pembrolizumab plus trastuzumab in trastuzumab-resistant, advanced, HER2-positive breast cancer (PANACEA): a single-arm, multicentre, phase $1 \mathrm{~b}-2$ trial. Lancet Oncol 2019;20:371-82.

15 Segura PP, Gil M, Balañá C, et al. Phase Il trial of temozolomide for leptomeningeal metastases in patients with solid tumors. $J$ Neurooncol 2012;109:137-42.

16 Kumthekar P, Grimm SA, Avram MJ, et al. Pharmacokinetics and efficacy of pemetrexed in patients with brain or leptomeningeal metastases. J Neurooncol 2013;112:247-55.

17 Boogerd W, van den Bent MJ, Koehler PJ, et al. The relevance of intraventricular chemotherapy for leptomeningeal metastasis in breast cancer: a randomised study. Eur J Cancer 2004;40:2726-33.

18 Arbour KC, Mezquita L, Long N, et al. Impact of baseline steroids on efficacy of programmed cell death-1 and programmed death-ligand 1 blockade in patients with non-small-cell lung cancer. $J$ Clin Oncol 2018;36:2872-8.

19 Mack F, Baumert BG, Schäfer N, et al. Therapy of leptomeningeal metastasis in solid tumors. Cancer Treat Rev 2016;43:83-91.

20 Morikawa A, Jordan L, Rozner R, et al. Characteristics and outcomes of patients with breast cancer with leptomeningeal metastasis. Clin Breast Cancer 2017;17:23-8.

21 Remon J, Le Rhun E, Besse B. Leptomeningeal carcinomatosis in non-small cell lung cancer patients: a continuing challenge in the personalized treatment era. Cancer Treat Rev 2017;53:128-37.

22 Rodrigues G, Hoshino A, Kenific CM, et al. Tumour exosomal CEMIP protein promotes cancer cell colonization in brain metastasis. Nat Cell Biol 2019;21:1403-12. 
23 Fares J, Cordero A, Kanojia D, et al. The network of cytokines in brain metastases. Cancers 2021;13:142.

24 Le Rhun E, Devos P, Boulanger T, et al. The RANO leptomeningeal metastasis group proposal to assess response to treatment: lack of feasibility and clinical utility and a revised proposal. Neuro Oncol 2019;21:648-58.
25 Chi Y, Remsik J, Kiseliovas V, et al. Cancer cells deploy lipocalin-2 to collect limiting iron in leptomeningeal metastasis. Science 2020;369:276-82.

26 Remsik J, Wilcox JA, Babady NE, et al. Inflammatory leptomeningeal cytokines mediate COVID-19 neurologic symptoms in cancer patients. Cancer Cell 2021;39:e273:276-83. 